\title{
NORTH-SOUTH ASYMMETRY OF GEOMAGNETIC ACTIVITY AND SOLAR WIND ELECTRIC FIELD
}

\section{G.A. Makarov}

Yu.G. Shafer Institute of Cosmophysical Research and Aeronomy of SB RAS, Yakutsk, Russia gmakarov@ikfia.sbras.ru

Geomagnetic activity asymmetry in the Northern and Southern hemispheres is studied. It is shown that the higher is the level of magnetic activity the greater is the asymmetry. It is found that the asymmetry in the hemispheres manifests itself in the 06-18-hour component of magnetic activity diurnal variation, while the asymmetry in the 00-12-hourly component is completely absent. The cause of geomagnetic north-south asymmetry is supposed to be meridional Pedersen current between high and middle latitudes in the ionosphere. A qualitative model of formation of asymmetry connected with the solar wind electric field is proposed.

Keywords: geomagnetic activity, Earth's magnetosphere, interplanetary magnetic field.

\section{INTRODUCTION}

It is common knowledge [Siebert, 1968; Wilcox, 1968] that geomagnetic activity level in the positive sector of the interplanetary magnetic field (IMF) in the Northern Hemisphere is higher than that in the Southern one, while in the negative IMF sector it is, on the contrary, higher in the Southern Hemisphere. The above papers deal with three-hour geomagnetic data [Siebert, 1968] or diurnal variations for certain periods [Wilcox, 1968]. They do not, however, examine the effect of magnetic activity level on the asymmetry.

To reveal the physical nature of the north-south asymmetry of geomagnetic activity, it is necessary to consider it across the entire dataset and in periods with different geomagnetic activity levels.

We analyze three-hour data on geomagnetic indices $a_{\mathrm{n}}$ and $a_{\mathrm{s}}$ for 1968-1975 and their daily means $A_{\mathrm{n}}$ and $A_{\mathrm{s}}$ [Geomagnetic Data, 1972-1976]. The entire dataset is divided into two classes according to the sign of the IMF sector structure from the Sun (positive sector) and to the Sun (negative sector). Each class includes four groups depending on the magnetic activity level. The first group comprises days with a daily sum $\Sigma K_{\mathrm{p}}$ equal to $0 \div 12$; the second, $13 \div 18$; the third, $19 \div 23$; the fourth, $24 \div 33$. The data are processed by the method of superposed epochs. As a result, for each of the groups we acquire three-hour data and their monthly values. 


\section{ANNUAL AND DIURNAL VARIATIONS}

Danilov [Danilov, 1998] shows that the excess of magnetic activity in the positive IMF sector in the Northern Hemisphere is equal to that in the negative IMF sector in the Southern Hemisphere. To identify the asymmetry, we utilize the following combination of geomagnetic indices $A_{\mathrm{n}}$ and $A_{\mathrm{s}}$ of the Northern and Southern hemispheres respectively. As a working variant $A$ we compute the sum of monthly averages $A=\left(A^{+}{ }_{\mathrm{n}}+A^{-}{ }_{\mathrm{s}}\right)$ where signs $«+»$ and «-» refer to positive and negative IMF sectors. For comparison, we take a control variant $K=\left(A^{-}{ }_{\mathrm{n}}+A^{+}{ }_{\mathrm{s}}\right)$. The findings for the four groups $\Sigma K_{\mathrm{p}}$ are given in Figure 1. It is seen that curves in the working variant $A$ are drawn higher than those in the control one $K$. Yet the difference between $A$ and $K$ increases with magnetic activity. In quiet periods $\left(\Sigma K_{\mathrm{p}}=0 \div 12\right)$, the difference between $A$ and $K$ virtually disappears.

Consider variations in $a_{\mathrm{n}}$ and $a_{\mathrm{s}}$ with the time of day. To do this, by using data on three-hour $a_{\mathrm{n}}$ and $a_{\mathrm{s}}$, we conduct the Fourier analysis and determine the amplitude $R$ and time $T$ of maximum diurnal variation. For the time $T$ we take the solar time of the Northern Geomagnetic Pole that differs from UT by $4.5 \mathrm{hr}$. In the solarecliptic coordinate system, the vector with the amplitude $R$ and maximum $T$ phase (in degrees) can be resolved into two components $a_{x}=R \cos T$ and $a_{y}=R \sin T$ aligned with the geomagnetic meridian and parallel respectively.

The working variant corresponds to the sum $\left(A_{\mathrm{n}}{ }^{+}+A_{\mathrm{s}}{ }^{-}\right)$; the control one, to $\left(A_{\mathrm{n}}{ }^{-}+A_{\mathrm{s}}{ }^{+}\right)$where the indices denote the Northern $(\mathrm{N})$ and Southern $(\mathrm{S})$ hemispheres and the IMF sector polarity. Numbers indicate magnetic activity levels: $1-\Sigma K_{\mathrm{p}}=0 \div 12 ; 2-13 \div 18 ; 3-19 \div 23 ; 4-24 \div 33$.

If geomagnetic activity is caused by ionospheric currents, then it is obvious that the $a_{x}$ component is driven by currents flowing along geomagnetic parallels; and the $a_{y}$ component, by currents flowing along the meridian. From the $a_{x}$ and $a_{y}$ components we form combinations of $A$ and $K$. The results are shown in Figure 2. Notice that for $a_{y}$ we take absolute values because this component has opposite signs in the positive and negative IMF sectors.

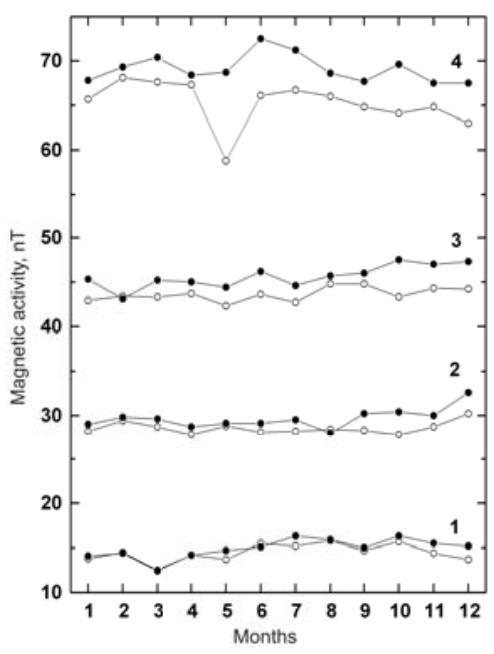

Figure 1. Annual variation of magnetic activity for the working $(\bullet \bullet)$ and control $(\circ-\circ)$ variants 

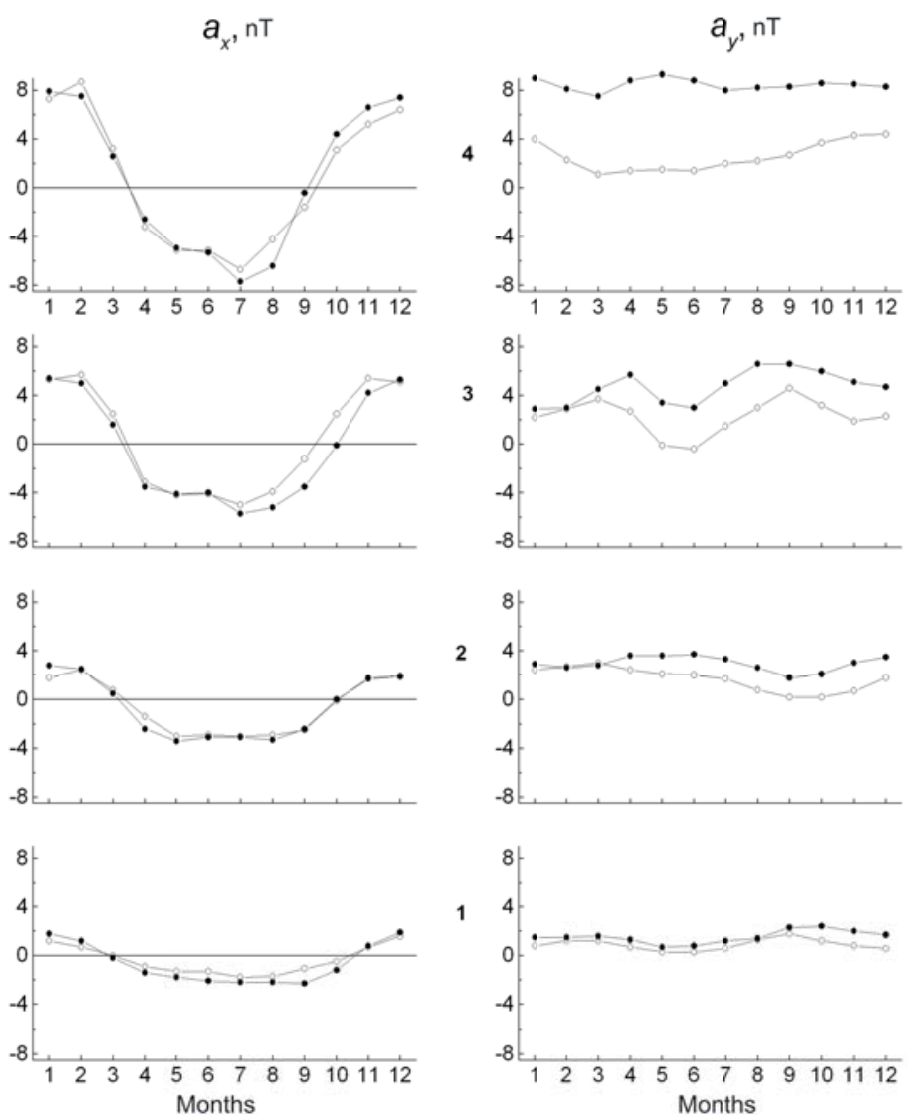

Figure 2. Annual variation in $0 \div 12$-hour $\left(a_{x}\right)$ and $6 \div 18$-hour $\left(a_{y}\right)$ components of the diurnal variation of magnetic activity for the working $(\bullet \bullet)$ and control $(\circ-\circ)$ variants. Numbers indicate magnetic activity levels: 1 $\Sigma K_{\mathrm{p}}=0 \div 12 ; 2-13 \div 18 ; 3-19 \div 23 ; 4-24 \div 33$

It is apparent that for $a_{x}$ the $A$ and $K$ variants are almost alike, whereas for $a_{y}$ curves of $A$ are much higher than those of $K$. This implies that the south-north asymmetry of magnetic activity is caused by ionospheric currents flowing along the meridian.

\section{INTERPRETATION OF THE EFFECT}

Siebert and Wilcox [Siebert, 1968] and [Wilcox, 1968] believe that Earth's hemispheres are more sensitive to geomagnetic disturbances when Earth's magnetotail is opposite in direction to IMF and magnetic reconnection can occur. This idea provokes two comments. First, in tail lobes the field has only the $B_{x}$ component. Consequently, the reconnection can take place only with the $B_{x}$ IMF component. However, Shelomentsev and Yoshizawa [Shelomentsev, 1977; Yoshizawa, 1986] show that the northsouth asymmetry of magnetic activity is associated with the IMF azimuthal component $B_{y}$. Petrukovich [Petrukovich, 2011] using Geotail satellite data has revealed that the IMF $B_{y}$ component significantly affects the magnetic field $B_{y}$ component in the plasma layer, which depends on the geodipole tilt angle. In [Cao et al., 2014], this result was confirmed through Cluster measurements. Accordingly, the magnetic field reconnection in radial direction cannot explain the asymmetry. Second, if it is granted that Earth's magnetotail and IMF do reconnect, it remains unclear how the reconnection energy is transferred to middle latitudes because tail lobes are connected with the polar cap through magnetic field lines. 
Shelomentsev [Shelomentsev, 1977] thinks that during periods of (+) IMF the morning-side auroral electrojet increases, and back currents in the Northern Hemisphere shift toward the equator; and in the Southern Hemisphere, to the pole. The author concludes that back Hall currents of the morning-side electrojet cause the north-south asymmetry. It is not correct since the currents flow largely along parallels, and their magnetic effect should manifest itself in the $a_{x}$ component. Figure 2 indicates that in this component the asymmetry in the hemispheres does not reveal itself at all. Referring to this figure, the asymmetry shows up in the $a_{y}$ component of the diurnal variation, i.e. it is generated by meridional currents.

Yoshizawa [Yoshizawa, 1986] notes that the asymmetry value varies with substorm activity. The author has established that the asymmetry value depends on the local magnetic time. Magnetic and substorm activities are dissimilar notions. Unlike substorm activity, magnetic activity does not necessarily vary in response to energy accumulation in the magnetotail.

We suggest the following qualitative pattern for the formation of the north-south asymmetry of magnetic activity. IMF penetrates into magnetotail lobes and generates an electric field $\mathbf{E}=-[\mathbf{V} \times \mathbf{B}]$ where $\mathbf{V}$ is the solar wind velocity, $\mathbf{B}$ is the tail magnetic field directed to Earth in the northern tail lobe and from Earth in the southern one. In the tail lobes, electric drift of plasma occurs (Figure 3). The drift velocity $\mathbf{V}_{\mathrm{A}}$ is seen to be directed from dawn to dusk in the working variant, and vice versa in the control one. At ionospheric heights, only ions drift, while protons stay put due to frequent collisions with neutrals. Accordingly, in the polar ionosphere there are dusk-dawn currents. This is consistent with the well-known fact that in the positive IMF sector in the Northern Hemisphere the dusk convection vortex expands into a large part of the polar cap [Ionospheric-Magnetic Disturbances, 1986]. This is equivalent to the appearance of electric current across the polar cap.

In the working variant, positive charges gather on the morning side of the polar ionosphere; negative ones, on the evening side. These drift charges amplify the existing electric field $\mathbf{E}$ generated by fieldaligned magnetospheric currents of zone 1 [Iijima, Potemra, 1976].
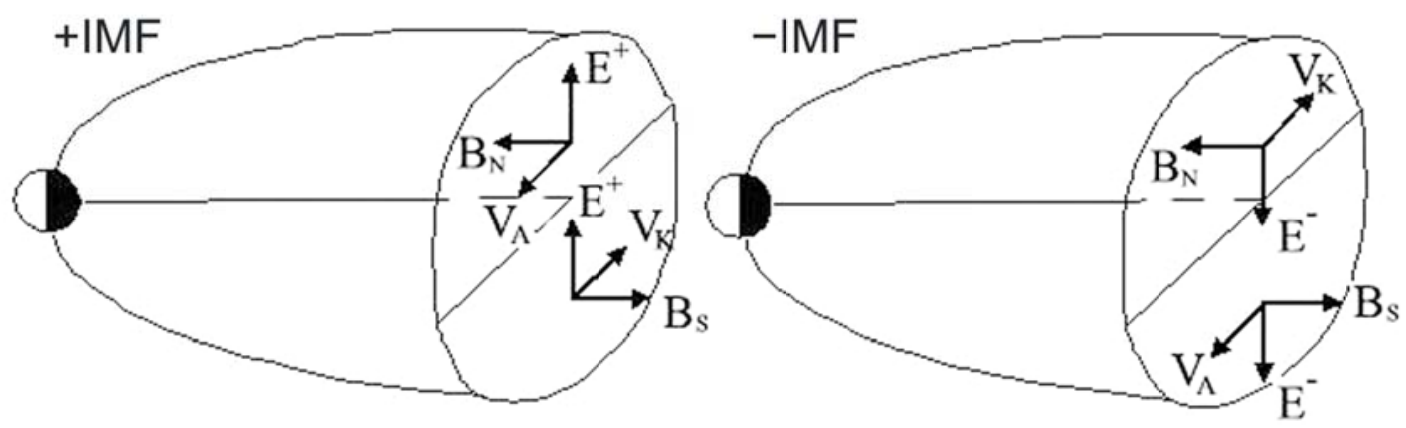

Figure 3. Diagram illustrating electric drift of plasma in magnetotail lobes in the positive $(+)$ and negative (-) IMF sectors. $\mathbf{B}_{\mathrm{N}}$ and $\mathbf{B}_{\mathrm{S}}$ is the magnetic field in the northern and southern tail lobes; $\mathbf{E}^{+}$and $\mathbf{E}^{-}$is the solar wind electric field; $\mathbf{V}_{\mathrm{A}}$ and $\mathbf{V}_{\mathrm{K}}$ is the plasma drift velocity in tail lobes for the working $A$ and control $K$ magnetic activity variants 
In the control variant, the drift occurs from dusk to dawn, and drift charges attenuate the field caused by currents of zone 1 . In the working variant there emerges an additional electric field between high and middle latitudes. This field exhibits auxiliary Pedersen currents flowing from high to middle latitudes in the dawn sector of the ionosphere and backward in the dusk one. The auxiliary Pedersen currents together with existing currents in the polar ionosphere close inflow currents of zone 1 and outflow currents of zone 2 that are likely to exist throughout the region from middle latitudes to the auroral zone. Thus, in the positive IMF sector in the Northern Hemisphere there arises an additional current system that includes field-aligned currents of zones 1 and 2, drift and Pedersen currents in the polar and mid-latitude ionospheres. During the negative IMF, a current system appears in the Southern Hemisphere. In the control variant, this current system does not develop because it is interrupted in the polar cap.

\section{CONCLUSION}

The main results of the study are as follows.

1. By using a large body of experimental data, we have confirmed the conclusion that in the positive IMF sector the level of geomagnetic activity in the Northern Hemisphere is higher than that in the Southern one, while in the negative sector it is higher in the Southern Hemisphere. In addition, we have established that the higher is the level of magnetic activity the greater is the asymmetry in the hemispheres. During quiet periods there is no asymmetry.

2. The asymmetry in the hemispheres appears in the $a_{y}$ component of the diurnal variation of magnetic activity, whereas in the $a_{x}$ component the asymmetry is completely absent.

3. An immediate cause of the north-south asymmetry is supposed to be meridional Pedersen currents flowing between high and middle latitudes in the ionosphere.

4. We have proposed a qualitative model of asymmetry formation. The solar wind electric field $\mathbf{E}=$ $[\mathbf{V} \times \mathbf{B}]$ penetrates into magnetotail lobes and generates here an electric drift of plasma. At ionospheric heights, this drift enhances the electric field across the polar cap and generates an additional electric field between high and middle latitudes. In this field there arise Pedersen currents responsible for the observed asymmetry of magnetic activity in the two hemispheres.

I began this study together with A.A. Danilov who passed away on December 24, 2011.

\section{REFERENCES}

Cao J.B., Duan A., Dunlop M., Wei X., Cai C. Dependence of IMF $B_{y}$ penetration into the neutral sheet on IMF $B_{z}$ and geomagnetic activity. J. Geophys. Res. 2014, vol. 119, pp. 5279-5285. DOI: 10.1002/2014JA019827.

Danilov A.A. North-south asymmetry of geomagnetic activity. Geomagnetizm i Aeronomiya [Geomagnetism and Aero-nomy]. 1998, vol. 38, no. 3, pp. 39-46 (in Russian).

Geomagnetic Data. IAGA Bulletin 1972-1976, no. 32, a-h.

Iijima T., Potemra T.A. The amplitude distribution of field-aligned currents of northern high latitudes observed by Triad. J. Geophys. Res. 1976, vol. 81, pp. 2165-2174. 
Ionospheric-Magnetic Disturbances at High Latitudes / Ed. O.A. Troshichev. Leningrad: Gidrometeoizdat Publ., 1986. 256 p. (in Russian)

Petrukovich, A.A. Origins of plasma sheet $B_{y}$. J. Geophys. Res. 2011, vol. 116, A07217. DOI: 10.1029/ 2010JA016386.

Shelomentsev V.V. Correlation between the north-south asymmetry and IMF y-component. Issledovaniya po Geomagnetizmu, Aeronomii i fizike Solntsa [Research on Geomagnetism, Aeronomy and Solar Physics]. Moscow, Nauka Publ., 1977, iss. 43, pp. 55-59 (in Russian).

Siebert M. Magnetic activity differences between the two hemispheres following the sector structure of the interplanetary magnetic field. J. Geophys. Res. 1968, vol. 73, pp. 3049-3052.

Wilcox J.M. Asymmetry in the geomagnetic response to the polarity of the interplanetary magnetic field. $J$. Geophys. Res. 1968, vol. 73, pp. 6835-6836.

Yoshizawa K. Effect of the IMF- $B_{\mathrm{y}}$ component on the North-South asymmetry of geomagnetic activity. Planetary and Space Sci. 1986, vol. 34, pp. 639-644. DOI: 10.1016/ 0032-0633(86)90041-3. 